\section{A prospective study of physician- observed concussion during a varsity university ice hockey season. Part 1 of 4}

\author{
Paul S. Echlin, M.D.
}

\author{
Elliott Sports Medicine Clinic, Family Medicine, Burlington, On- \\ tario, Canada
}

I had negative feedback from the coach who thought that the study was a waste of money... he didn't want his players to get assessed in fear that they would be declared as concussed and advised not to play... the players didn't want to be declared as concussed since it would limit their playing time.

HCEP OBSERVER

While it appeared most players and officials were open to the project, there was an air of "us" versus "them" and I could tell they were willing to partake as long as it didn't affect the outcome of the game, season, career... The coach/team official was rarely directly involved and tended to avoid conversing with us.

HCEP OBSERVER

The 2011-2012 Hockey Concussion Education Project (HCEP) used physician-led direct clinical identification and diagnosis methodology, advanced MRI scanning (diffusion tensor imaging, MR spectroscopy, and susceptibility weighted imaging) before and after the season, as well as sequential testing at 3 time points after injury. The MRI component was utilized to investigate objective tools that could be used to improve the sensitivity of current clinical and neuropsychological methods of diagnosis and return-to-play decisions.

The 2011-2012 HCEP was developed, designed, and implemented as a follow-up to the 2009-2010 HCEP study $^{2-5}$ that investigated concussion incidence, return to play, and education in 2 fourth-tier men's junior ice hockey teams during 1 competitive season in Canada. The 2009-2010 study demonstrated the underreported incidence of concussion, which was 7 times greater than had previously been reported in the literature. In addition, observations from different levels in hockey culture demonstrated social and cultural resistance to change in my editorial. ${ }^{2}$

This editorial will review comments and responses from study observers -14 physicians and 10 nonphysicians - to the primary investigator that occurred throughout the 2011-2012 HCEP. The editorial summarizes observations about the roles of different people around young athletes as they make their health care decisions.

\section{The Coach}

The young athlete is often caught between competing demands of the adults around them. Young athletes sometimes make decisions based on the adult whom they perceive to have the most influence on their success, and also whom they wish most to please for a variety of reasons. In many cases, the coach is perceived to be the adult with the most influence on the success of the athlete. The coach determines the amount of time the athlete will play during a specific game or season.

To improve concussion prevention and care, the coach is central to potential social change:

I think the most critical change is education of players and coaches. I do not believe players understand the risks of playing with concussion symptoms. In the heat of battle I am often concerned that coaches do not have the best interests of their players at heart...coaches should be willingly pulling players after "big hits" or when concussion may be suspected. I think there is still a lot of old culture in hockey that says "be tough and get back out there-the team needs you." This culture often starts from the coach.

HCEP OBSERVER

I find that the players and coaches often downplay the symptoms in an effort to get the athlete back into action. I think it relates to the culture of hockey. Players are scared to be seen as weak and almost always want to play. Coaches expect their players to "shake it off" and "take it for the team" and get back on the ice. I think coaches fail to admit the significance of the symptoms.

HCEP OBSERVER

One HCEP physician (neurologist) observer, who is also a hockey player and team physician, experienced ongoing resistance to his participation in the study. The following report reveals how a coach ignored his diagnosis and his recommendation that the player sit out. This was followed by the coach's request that he be removed from observation of the games. The report reveals how a coach (and trainer) can resist treatment of suspected concussion; the physician observer said:

We watched the first period and the player sustained two hits separated by a couple of minutes apart with less than 10 minutes left in the period. He seemed a bit slow skating up the ice after the second hit. We decided to pull him out and check him over after the period was over. His Sport Concussion Assessment Tool-2 (SCAT2) objective score was positive, but most significantly he self-reported feeling dizzy after the second hit when skating up the ice. He also stated that he felt dizzy in the dressing room when we assessed him.

We advised the player to sit out, but his coach came in and confronted him and said, "What's the problem? You're not dizzy are you? Why don't you go out for a couple of shifts in 
the second period and skate it off?" I observed the player was now in a very difficult situation: he was intimidated and was being asked to make a difficult choice. Of course he was going to say he felt okay. We then watched him in the second and third period. He was clearly one of the best defenseman on the team, but just wasn't playing at a top level. At the beginning of the second period he took a check and went down. It wasn't a particularly strong hit. Next, he was back checking and when his skates were crossing over, they got tangled up and he fell without any body contact. I think given the level of play this young man is at, that this was evidence of concussion. Furthermore he was on for the first and third goals that the opposing team scored and it wasn't just being on; he actually made mistakes that resulted in the goals.

At the end of the third period, I spoke with the player and the trainer and said that he should not play until he was formally evaluated and underwent the formal return to play protocol. I was dismayed to see that he played the next evening.

After returning from the road trip the trainer was questioned about his decision to overrule the direct medical diagnosis of concussion and the decision of the neurologist to restrict the player. He was also questioned about the significant risk that he had placed upon the player. The trainer responded that he and the player did not understand the decision and that most of the team did not trust the neurologist. He requested that the physician no longer be used to cover any more games.

In some circumstances, a culture of intimidation practiced by coaches was extended to physician observers working on the study. Hockey culture is familiar with coach admonishments to play through injuries, or to "suck it up." During this study a coach said, "Unless something is broken I want them out playing." Intimidation was also used to try to preempt behaviors certain coaches did not desire for the team, including the following exchange with an HCEP physician:

Physician: How is the study going for the team to this point? Are there any concerns about the protocol?

Coach: No problems now. We will see when you make a concussion call.

Another significant incident occurred when a player suffered what the HCEP game physician assessed as a minor concussion during the 2 nd period of a game. Nevertheless the player was allowed to play the 3rd period. At the end of the game, the player was still reporting symptoms of feeling "iffy" and "off" and slightly dizzy; therefore, another clinical evaluation was performed to confirm the diagnosis of a concussion and to recommend exclusion from upcoming games.

The coach's response to the physician's assessment at the end of the game was "that is not a fair assessment." However, the coach did acknowledge that the player's persistent symptoms were of concern and that the player "had the rest of her life ahead of her." The coach then asked the physician, "On whose authority do you restrict the player from the game?" This challenge came despite the fact that the coach was well informed about the quality and nature of the care provided to her players involved in the HCEP and a personal history of her own career ended by multiple concussions.

When an HCEP physician arrived for a game between the same teams the following evening, the coach had de- cided that the player was fine and was going to play. The physician was able to communicate her concerns with the player and the trainer during the first period intermission. The player initially seemed receptive to the recommendations of the physician, including sitting out the remainder of the game in the hopes she would be able to play safely the following week. However, she returned to play against medical advice, stating that she felt $100 \%$.

The trainer was asked by the physician to speak with the coach after observing the return of this player for the 2nd period. In the interests of protecting the athlete, the physician wanted to make sure that her recommendation of athlete restriction was clearly communicated to the coach. In response the trainer looked very concerned and said the coach would be irritated by this request because the coach did not want the research study to "interfere" with the team. The coach refused to speak with the physician, and the athlete played the third period.

The response from a senior HCEP physician concerning this second instance of a coach overriding a medical diagnosis was: "Interesting gap between theory and practice... The athlete's and coach's decision to return to play the next day despite incurring a minor concussion reflects what occurs thousands of times every day. Not ideal, but reality-based. I think that we should make note of the event and move on."

The senior HCEP physician discussed this incident at length with the HCEP primary investigator (PI). The coach's decision to override the direct medical diagnosis was unacceptable. The senior physician appeared to understand the risk at which the coaches were placing the player, and how they were compromising the protocol of the study. The senior physician then took the initiative to talk to the coaches concerning the seriousness of their actions and to discuss the possibly life-altering or lifethreatening consequences for the athlete.

After this second incident of a coach overriding a direct medical diagnosis of concussion, there were no other observed and reported events. This may represent a learning curve that occurs when new protocols are introduced. Previously accepted behaviors such as making a returnto-play decision after a suspected injury are being challenged in the study protocol.

At the conclusion of the study one of the coaches made the following comment:

I have an issue with the protocol of the study. It is mainly with the role of the observer and the doctor. For example, when there is a hit during the game, both the observer and the doctor have seen the hit in question. Following the hit, they will draw their own conclusions as to what the result of the hit will be. Remember, they are not the ones taking the hit, only the ones observing it. Following that, the observer and doctor will visit the player and ask him questions. These questions are asked in order to verify that the player indeed has a concussion. To me and to our players, it seems that the study is heavily weighted towards finding a concussion.

What's the solution? I feel that you should take the doctor completely out of the game. He or she should not watch the game. Instead, have the observer watch the game and record any hits/plays in question. If a player has been hit then the medical trainer takes him into the room for the doctor to make an assessment. This would be a completely objective assess- 


\section{Editorial}

ment from the doctor. Following his assessment he can review his findings with the observer.

As you may know, watching something live at full speed, especially a collision in hockey, can look far worse than what it actually is. Much the same as that what might appear to be an irrelevant hit could be much more damaging than at first glance.

These comments demonstrate current practice in sports culture. The physician is not part of the sporting environment and should not be consulted to make a diagnosis unless called upon by an individual with less clinical training such as the coach or the trainer.

The coach who made the above statement also assumes that the physician observer has no personal sport expertise or experience and may be biased toward diagnosing concussions in players. In this HCEP study, all physician observers had strong sports medicine and team physician backgrounds, and more than half of these individuals had played the game.

The independence of the well-qualified physicianobserver allows him or her to observe the probable concussion, and to make a diagnostic decision without interference from the individuals with a stake in the outcome of the game. It is my observation that the independent physician observers were empathetic toward the athletes' desire to play and initially were very cautious about making the decision to diagnose and restrict a player secondary to an acute concussion diagnosis.

\section{The Physician}

Despite the interference experienced from the team, I don't feel as though my determination of whether the player was concussed or not was affected.

I think that I was at times conscious of the potential disruption/interference that removing players continually for SCATs could cause... This was particularly true for the borderline hits. If I'm honest, I did at times possibly avoid screening a minor/ borderline hit because I wanted to be careful not to "lose the room" so to speak.

\section{HCEP Physician ObSERVER}

The above statements were made by one of the experienced and dedicated HCEP physician observers and a former junior-level hockey player, describing the difficulty of his responsibilities during the study.

The team physician's first duty is to protect the patient/athlete by providing independent medical care focused solely on their short- and long-term health. Ideally the player's medical management should be devoid of environmental pressures to remain in play regardless of sustaining an injury. The struggle with team-related bias occurs in the relationships that develop between the physician, players/trainers/coaches, and team administration. Examples of the HCEP physician's observations of interactions are reported below:

My suspicion for the goalie was quite low and if I was not doing the study I would not have done a full SCAT on her, but in the context of the study I felt I still should, even though it didn't change my decision. I think with a low suspicion it is quite a big deal to pull a goalie and the girls might not be so willing to participate if we are pulling key players that we normally wouldn't pull...
Physicians inexperienced in evaluating concussions can have difficulty under the current identification and diagnostic protocol. The team physician often experiences both bias and cultural resistance when determining diagnosis and return-to-play decisions. A senior physician (a team physician for 20 years) admitted after observing his first game from an elevated position strictly looking at head contact under HCEP protocol, that he "felt guilty" that he really had not been paying attention to possible concussion incidence in the past. Often the observed explanation for nonadherence to the protocol by the physician or trainer was made to appease the player and coach, despite knowledge of the purpose of the protocol and medical responsibilities.

\section{The Athlete}

The reluctance of athletes to self-report a possible concussion and their observed inclination to mask concussion symptoms is a major obstacle to concussion identification. This reluctance to report is often a result of their fear of losing playing time during the recovery process.

One player sustained a hit that caused obvious symptoms. He went directly to his team's bench, and was observed to hide behind his back-up goalie and other teammates for a couple of shifts before returning to the bench for a regular shift. The observer thought that it was because the player knew he/she was being watched: "I had the feeling that the players were not too happy to see the doctor and myself. Having played junior hockey I can relate to that. They don't want to be pulled out of a game for symptoms that may seem minor at the time."

The following is a description of an athlete and trainer who attempted to conceal symptoms in an effort to continue to play despite sustaining a probable concussion. The unfortunate result was a prolonged period of recovery:

At the game the player took an elbow to the head. She got up immediately, and although she felt some mild symptoms at the bench (nausea), she noted that she had felt that before and did not have any prolonged symptoms or diagnosis of a concussion. She was not assessed by the study observers at that game, and she played next evening's game. She continued to feel sick over the next few days/weeks... Her symptoms initially mostly consisted of a headache, but she also experienced nausea with minimal activity (walking to classes) and after a couple of weeks noted increased sensitivity to light. She was approached by the trainer who did objective concussion testing (SCAT2), but didn't notice any abnormalities except for some symptoms that they both attributed to having been sick...the player specifically asked the trainer not to disclose to the doctor her current symptoms so that she could continue playing and the trainer obliged.

HCEP PHYSICIAN OBSERVER

While it is difficult to quantify the inclination of athletes to not report injury in order to keep playing, physician observers in this study did consistently report resistances by athletes to reveal possible concussions.

\section{The Trainer/First Responder}

I saw the goalie take head contact from the opposing player (she got run), then she fell down and her head took a second 
hit on the ice. She did not pop up and I went out to evaluate. I asked her if she was ok and she replied that she had a small headache, so I told her to stay down for a bit. Eventually I allowed her to stay in the game without an appropriate physician evaluation.

TRAINER TO THE HCEP PI

(This trainer, who was part of the study, had recognized the possibility of a concussion, but proceeded to bypass the physician evaluation)

The primary responsibility of the trainer is as a first responder concerning the injury or other health issues of the athlete. A trust relationship is often formed between athlete, trainer, and team administration. If the trust is disturbed by a perception of the players or other members of the team that the trainer is unnecessarily restricting an athlete, the trainer's position and ultimately his/her employment can be jeopardized. The HCEP independent physician observer assumed the responsibility of initial concussion identification and diagnosis, which removed this responsibility from the trainer.

Conflicts arose during the study that disrupted the relationship of trust between the trainer and the team. In 1 case, a player was identified with a concussion by the physician and the trainer. The player was examined the next day by the physician and the trainer. The player admitted to symptoms, but said that they existed prior to the hit, including "migraine" symptoms that had not been previously discussed. The trainer later admitted to the HCEP PI that he "talked the physician involved into returning the player to play because the player denied symptoms and the team needed him on the upcoming road trip." This same player was diagnosed with a concussion 2 weeks later by a neurologist, and the same trainer returned the player to play, overriding the decision by the neurologist:

I thought that initially, the training staff was very involved and excited about participating, but eventually this waned (by the end, the male trainer often times would not have his radio ear piece on, but would have to only talk to him at intermissions, or get his attention by other means if during play). I think that the trainers were quite close to the players and that at the time probably found it difficult to participate in certain instances as I'm sure they felt like they were being complicit in withdrawing a player against their will. As an independent physician with no personal connection/relationship, this job was much to easier do.

HCEP PHySICIAN OBSERVER

\section{Athletic Administration and Medical Researchers}

Prior to the initiation of the 2011-2012 HCEP, multiple institutions were invited to participate in this fully funded multisite study. Remarkably few institutions demonstrated interest. The telling reason given by 3 of the prominent institutions was that they did not believe the coaching staff would accept the direct monitoring and independent medical decision-making concerning their players.

At another level, the culture of resistance was revealed in the following letter from a grant evaluator:

It is very unclear how a physician sitting in the stands (not affiliated with the team) will go to the bench area after identifying a player with a "suspected" concussion, perform an evaluation on an athlete, and then determine whether they would return to play. This is absurd and I cannot envision any varsity hockey team program permitting this during competition. It would be useful to have letters of support from the athletic director, coach, current team physician, and athletic trainer.

These two examples represent the resistance encountered within the sports administrative and research communities. There is an apparent lack of independent, cooperative, public health initiatives to confront the issue of directly defining concussion incidence and enacting effective prevention-based solutions. One of the primary obstacles may be the fact that the sports that have the highest incidence of concussion also have the most significant public resistance to getting rid of violence.

\section{Parents of the Athlete}

The parent has a primary role in the development of young athletes' attitudes toward competition, excellence, and health. Parents of young athletes face multiple responsibilities. One responsibility is to support their child's athletic success through giving time and financial assistance. Another responsibility is to protect the long-term mental and physical health of their child. The priority to protect their child's long-term health can sometimes be overlooked as parents weigh the developmental values associated with sport and their children's accomplishments and achievements. Parents invest time, money, energy, and good intentions in their children's extracurricular sports. Often, a family's leisure time revolves around amateur sports schedules. When there is injury that requires treatment and rest, the rhythm of family relationships and the activities during family time are affected. Consider this comment made in a doctor's office by a father to his 11-year old concussed son: "What are we going to do now with our time?"

The PI received the following poignant e-mail from a distressed "hockey mom" with a description of her son's experience:

My 13 year old son is a small guy, weighs $90 \mathrm{lbs}$ and is just over 4.5-ft tall. Unfortunately he has had his share of concussions, two diagnosed concussions in the last calendar year alone. The last one happened on November 20 and he just returned to play on January 9. We wanted to give him a long healing period, despite the looks of disdain from other parents who just don't understand. They all say that they hope he feels better, but he needs to get out on the ice and play.

My husband and I would continue to go to every game while our son was off and I could not get over the amounts of injuries I saw to kids on the opposing team that would sustain an injury that would require the trainer to go out for and it was obvious that these kids had their "bell rung" but yet they were out the next shift. It frustrated me so much, that I started to yell to keep them off the ice. Our hockey association does not have a concussion regulation (if that is the right thing to call it) but I have heard some rumblings that the board would like to investigate it more and possibly adopt something in the near future.

I have come to realize that I have found a new passion in life and want to bring the seriousness of concussions to the forefront, not only in minor hockey, but all sports involving our youth. My son has realized that his dream of taking hockey to higher levels will soon have to end due to his past concussions, but it does not have to be that way for all kids. 


\section{Editorial}

The PI also received a telephone call from a distressed parent of an elite 14-year-old hockey player who had witnessed his son sustain a hit to the head against the boards. He observed his child getting up slowly and gradually making his way to the bench. After he arrived at the bench, he was briefly approached by the coach, and then was left on his own in a slumped-over position.

The father knew that something was wrong with his son, so he ran down to the bench and took him back to the dressing room in the middle of the game before his next shift. The next day the child was medically diagnosed with a concussion. This child was fortunate that his father took it upon himself to protect his child's health and was not intimidated by the hockey culture.

This chilling statement was made by the father of one of the athletes from the 2009-2010 HCEP study:

One answer is simple: that we put our desire of being able to brag about our children's accomplishments ahead of their welfare. The children just want to please us and they see the response they get from getting a goal, or laying on the best "hit" of the game. So we reinforce this aggressive behavior and over time it becomes natural for our kids to go out and nail the opponent in order to get the applause and praise from us, the coaches, and spectators (and they get more ice time as a reward, which is like a drug - they have to have it).

Because of this misaligned thinking, our son is now no longer able to play the sport he grew up with and loves. And we have to live with the fact that we pushed him to be aggressive and to hit and be hit so that we could feel proud and have others tell us how great he was. We had the choice and we made the wrong decision. We loved our time at the rink and our time with him, but we now question whether we could have enjoyed the time with our son without putting him in danger.

I should have been smarter, somehow (his voice thickening with emotion). So yeah, I have total guilt and I will for the rest of my life on this one.

\section{Opinion Statement from the Author}

The pressure to win the next period, game, or series is an important and overriding factor that blinds many of those who are responsible for protecting the health of our young athletes. Hockey and other contact sports need to be respected as a game, not as a potentially life-and-death battle that places participants at needless risk for future long-term disability. ${ }^{2}$

The win-at-all-costs philosophy is dangerous when it filters down to the minor hockey player who emulates his/her hero by not admitting to a concussion, or complies with their coaches or other adult leaders who resist reporting concussion for fear of having to take that player off the playing surface.

Jeff Blair in his piece in the Toronto Globe and Mail wrote about the injury suffered by Sidney Crosby and the public message that it sent: ${ }^{1}$

Mr. Crosby's concussion is a telling moment for hockey at all levels. He is the game's most important player. His team's future-even its survival as a franchise-depends on his health. If even Mr. Crosby can receive a crushing and direct blow to the head without receiving medical attention, what athlete-of any age, at any level-can be sure of receiving proper care?

A change in the culture of sports concerning the seriousness of brain injury is fundamental to reducing the incidence of this type of injury, and to improved identification and treatment. Our sporting culture has a characteristic resistance to change, particularly when it appears to threaten individual freedom. From the safety of our spectator's seat, when there are no personal repercussions to our own lives, we may feel justified in supporting individual choices about health risks. But it is too late to reevaluate this choice after a young person sustains a brain injury. It is the responsibility of all adults to protect our young people from long-term disability caused in amateur sports.

The statement below was made by a physician who had observed several games at different time points of the study:

At the end of the game, I was approached by a player with a recent concussion diagnosis and protocol restriction. He stated he was feeling back to normal and "totally fine." He then asked if he might be able to play Game 3 . The coach also spoke to me and asked if it was possible that he "might be able to be medically cleared to return to play?" I advised both of them that the answer was no and reiterated the return to play protocol, etc. The trainer, talking to me afterwards, stated he had already gone over this with the player and coach as well.

I spoke with the coaches after the game and said that we appreciated their cooperation. They responded in kind. The trainer was also thankful for the participation. I must say that overall, I noted a change (improvement) in the coaches attitudes over the last 2 weekends compared to previously. I'm not exactly sure what went on behind the scenes to accomplish that, but they did seem more willing to participate.

Another physician reported the following statement from one of the coaches concerning the experience at the end of this study: "The variance of concussion presentation that I had not previously known about was interesting: from the obvious mechanism and presentation to nonobserved self presenters, each athlete presenting with different sets of symptoms and symptoms that last for different lengths of time." It is the belief of the author that the statements by these two physicians demonstrate a positive shift in team leadership on this injury as a result of this study. This attitude shift is different from initial rejections of the study, resistances to physician diagnosis, and lack of cooperation between coaches, trainers, and doctors.

The social resistance to change concerning concussion identification and treatment is evident in the fact that despite significant efforts to educate teams and improve their care, in the third-to-last game of their season a player and coach in this study still sought to return to play immediately after a concussion.

Education is vital to decrease the incidence of concussion and improve treatment. Education and positive action are everyone's responsibility. To overcome cultural inertia concerning the growing knowledge about concussion, and the too-frequent inaction by supervising adults, we must look primarily toward educating the next generation of coaches and parents.

The priority must be placed on the short- and longterm health of each individual athlete over the outcome of any particular game or practice. Specific responsibility 
must be placed on the medical professionals working with the team coaches, administrators, and trainers to provide unbiased, independent, and prioritized care of the athlete. Until there is a breakthrough in our understanding of all aspects of concussion, each concussion should be treated very conservatively by trained medical specialists. We can no longer afford to treat this serious brain injury with the cavalier attitude that has been demonstrated in the past. The games that we play and want our children to play must evolve with the advancement of medical knowledge concerning concussion. This "silent epidemic" is significantly affecting the future of our youth, and at what price: to entertain us, or to win the next game? (http://thejns.org/doi/abs/10.3171/2012.9.FOCUS12287)

\section{Appendix}

\section{Neutral Observer Exit Questionnaire Comments}

Below are 4 of the questions and responses from the 2011-2012 HCEP physician and nonphysician observer exit questionnaire.

1) Do you think that the addition of an independent trained observer to the mandated team physician and trainer at home games and the trainer at away games would improve identification/diagnosis of possible concussions?

-Yes, the fast pace of the game means that it is easy to miss clinically relevant contact. I think the addition of an independent observer who is there solely to observe for head contact/concussion would certainly improve the identification of more potential concussions, which may have otherwise been missed because the play was missed by the MD/Trainer, who were busy doing something else. I think that it certainly provides a better overall view of the entire ice surface, particularly the near boards on the far end of the ice. Overall, however, the view is still not perfect, and a second observer on the opposite side is needed.

-Yes, because their focus would be on that single role whereas the physician and trainer have others. The observation point from the opposite blue line is a key idea as well. Ensures all areas of the ice are covered.

-Yes, a third party observer would be beneficial for concussion identification, as I believe the team dynamics/politics could possibly affect the perspective of a team doctor or trainer.

$-\mathrm{I}$ found the independent observer to be extremely useful and helpful to me.

-Yes. Having an extra set of eyes is a good way to ensure that possible concussions are not missed. Spreading out in the rink and adding a view from a different angle can add a great deal.

-Yes. Having a strategically placed independent nonphysician observer and physician observer helped greatly. It allowed us to see the entire game and minimize blind spots or miss contact potentially resulting in concussions behind the play.

2) What changes (e.g., protocol, education, penalties) do you think would assist with improved team compliance (physician, team, coach, and trainer) for reporting concussions?

-Mandatory $\mathrm{pre} / \mathrm{mid} /$ postseason education sessions for players/ coaches/trainers.

-Despite the extra paperwork, having trainer fill out "incident reports" for all concussions or other injuries and have them reviewed by MD who can decide if they warrant a further follow-up visit.

-I think more education for the coach is necessary (mainly the men's team one). The coach mentioned to me that he thought this study was a big waste of money, so he clearly didn't see the value of it. If the coach was more on board, I believe the players would be more likely to comply too since they wouldn't feel any pressure to play while concussed.

-Education; an understanding of the damage that can result; provide the athletes and coaches with real case studies illustrating this damage.
-Education, but best started young so it's accepted and automatic, with endorsement from star athletes. Heavier penalties, including penalties even if it didn't appear intentional to deter players from hitting so much in the first place. Coaches and trainers should have repercussions based on ethical obligations of their professions.

-Because of the great variety of venues, levels of competition, and varieties of supervisory personnel, protocols are often difficult to design and enforce. They should remain uncomplicated. I agree with the examination of the athlete away from the coach or team trainer. I prefer repeated attempts at education and peer and culture support to penalties, which should be considered only in the most blatant abuses.

-I think the most critical change is education of players and coaches. I do not believe players understand the risks of playing with concussion symptoms. In the heat of battle I am often concerned that coaches do not have the best interests of their players at heart. This results in pressure being applied by coaches to continue in the sport even if concussion may be present. The opposite should be occurring - coaches should be willingly pulling players after "big hits" or when concussion may be suspected. I think there is still a lot of old culture in hockey that says "be tough and get back out there-the team needs you." This culture often starts from the coach.

-Increased education on short- and long-term impact of concussions.

-Education - "pay now or pay later"- understanding that early intervention means less time spent healing.

3) Did this study force you to look at concussions in a different way from your previous experiences when you attended or provided coverage for collision based sporting events? If so how?

-Yes, the biggest difference for me was how I watched the game looking at contact first as opposed to watching the play.

-Yes, now I see concussions as the serious brain injuries that they are, whereas before I didn't necessarily think they were that big of a deal. I just assumed it was a little bit of a headache that would go away no matter what you did (even if you kept playing). I no longer think that the player who "takes one for the team" and continues playing even though he/she is potentially concussed is a hero. In fact, I think he/she is uneducated. Now when I see a fight break out, or a player get hit in the head, I immediately wonder if they realize that they may be concussed. I hope that they know the signs of a concussion and are smart enough to stop playing and get the treatment they need so that they don't end up with permanent brain damage.

-Yes, I have become more aware of the potential long-term consequences of concussions. Players are continually getting stronger and faster and equipment is getting lighter, so will only become more important in the future.

-Yes, from a more neutral standpoint as I did not know the players/teams.

-From a hockey standpoint, yes. It encouraged me to follow the play after the puck specifically, rather than the actual game. The data sheet we recorded on also provided me with new ideas on what to watch and how to watch the game from a concussion standpoint.

-The observation experience was very intense. It was important to focus on the task and not get distracted when monitoring team games independently.

-Yes, I've realized it's not just "ringing your bell" and that you shouldn't just "shake it off." These were the messages I received throughout my athletic development and career. I knew more recently that these messages were not correct, but didn't understand the relevance of multiple smaller hits to the head. And the long-term effects of multiple head hits are shocking, and truly scary. I hope this information gets out to the mainstream population, and the rules and regulations help guide a new culture of head safety.

-No. In the last several years I have occasionally been frustrated by the attitudes of some of my colleagues and team officials but overall this exercise reinforced my beliefs regarding concussion.

-It definitely made me more proactive in seeking out concussions in the events I cover. 


\section{Editorial}

-Yes, reading about their severity was informative.

-Yes I had to overanalyze every check and hit.

4) In addition to direct neutral observation and diagnosis, what other method would you suggest to capture the true incidence of concussion?

-The players should be asked to fill out a questionnaire at the end of the season (probably best if it is anonymous) to ask them if they believe they sustained a concussion, or tried to hide it at all during the season. game?

-Anonymous self-reporting of symptoms by players after each

-Self-reporting is likely the only practical way to catch subtle injury. Pre- and posttesting of every player.

-More high-tech observation using multicamera observation with near-instant playback capability would enhance the screening capability of the study. I recognize the financial implications of this suggestion.

-Using video recordings and having an independent $\mathrm{MD}$ review each game within 24 hours and then discuss with team MD whether certain players were assessed during/following the game based on hits that occurred.

-Video camera replay to be reviewed by the independent observers and medical staffs.

-Video camera to see exactly where the point of contact was.

-Film the game and review with a record of the time of the game at which each concussion occurred and also the time at which minor hits/falls occurred that could have resulted in a concussion.

-More education in general for all people involved, players, parents, coaches, trainers, so that they can self report or offer a supportive social environment that is open to self reporting.

-Prescreening using concussion software as well as an information session for the athletes prior to the start of season would be beneficial. Also, any player that has had prior concussion should be monitored.

\section{Disclosure}

Funding for this work was provided to the HCEP and the HCEP PI (Dr. Echlin) by the Ontario Trillium Foundation, the Dave Irwin Foundation for Brain Injury Recovery, the Ontario Neurotrauma Foundation, and Air Canada. Dr. Echlin is a fellowship-trained Primary Care Sports medicine physician, who has been a junior-level hockey team physician from Major Junior OHL and
NCAA Division I, to the fourth-tier junior level for the past 10 years. Dr. Echlin is also the primary investigator of the games described by the HCEP.

\section{Acknowledgments}

The author would like to acknowledge the contributions of the University of British Columbia MRI center and all of the associated researchers and employees, especially Ms. Trudy Harris and Ms. Linda Chandler. The author acknowledges the editorial work of Kim Echlin, Ph.D., University of Toronto, Ontario, Canada. The author also acknowledges the players and staffs of two deidentified Canadian Interuniversity Sports varsity hockey teams for their participation in the HCEP, and would also like to acknowledge the participating physicians, observers, and volunteers for their contributions to the HCEP.

\section{References}

1. Blair J: Blind to what millions saw. Globe and Mail. January 13, 2011. (http://www.theglobeandmail.com/commentary/ editorials/blind-to-what-millions-saw/article561783/) [Accessed October 23, 2012]

2 Echlin PS: Editorial. Concussion education, identification, and treatment within a prospective study of physician-observed junior ice hockey concussions: social context of this scientific intervention. Neurosurg Focus 29(5):E7, 2010

3. Echlin PS, Johnson AM, Riverin S, Tator CH, Cantu RC, Cusimano MD, et al: A prospective study of concussion education in 2 junior ice hockey teams: implications for sports concussion education. Neurosurg Focus 29(5):E6, 2010

4. Echlin PS, Tator CH, Cusimano MD, Cantu RC, Taunton JE, Upshur REG, et al: A prospective study of physician-observed concussions during junior ice hockey: implications for incidence rates. Neurosurg Focus 29(5):E4, 2010

5. Echlin PS, Tator CH, Cusimano MD, Cantu RC, Taunton JE, Upshur REG, et al: Return to play after an initial or recurrent concussion in a prospective study of physician-observed junior ice hockey concussions: implications for return to play after a concussion. Neurosurg Focus 29(5):E5, 2010

Please include this information when citing this paper: DOI: 10.3171/2012.9.FOCUS12287. 